\title{
A DESCENTRALIZACÃO NA VIGILÂNCIA SANITÁRIA: TRAJETÓRIA E DESCOMPASSO
}

\author{
DECENTRALIZATION IN HEALTH SURVEILLANCE SERVICES: BACKGROUND AND MISMATCH
}

\author{
LA DESCENTRALIZACIÓN EN LA VIGILANCIA SANITARIA: TRAYECTORIA Y DESCOMPAZ
}

\begin{abstract}
RESUMO
Este trabalho descreve as características do processo de descentralização no Sistema Único de Saúde (SUS) e na vigilância sanitária, quanto à relação entre as esferas de gestão: centralização e descentralização. Utilizou-se uma abordagem analítica com foco na relação entre as esferas governamentais como fator constitutivo da gestão de uma política social. Os resultados indicam que a vigilância sanitária desenvolveu um processo lento e irregular de descentralização, que expressa claro descompasso com o mesmo processo no SUS, em relação à estrutura institucional e a estratégias de pactuação da relação entre as esferas. Conclui-se que as dificuldades enfrentadas no processo de descentralização estão relacionadas também às tensões advindas de diferentes projetos políticos que coexistem nesses espaços institucionais.
\end{abstract}

Palavras-chave: Descentralização, Sistema Único de Saúde, Vigilância Sanitária.

Regina Lucena - regina.lucena@ig.com.br

Professora Adjunta da Universidade Federal do Recôncavo da Bahia, Cruz das Almas, BA, Brasil.

Artigo submetido no dia 07-09-2014 e aprovado em 10-04-2015.

DOI: http://dx.doi.org/10.12660/cgpc.v20n66.32967

\section{ABSTRACT:}

This paper describes characteristics of the decentralization process in the Unified Health System (SUS) and health surveillance, with regard to the relationship between their management levels: centralization and decentralization. An analytical approach was used that focused on the relationship between levels of government as a constituent factor in the management of social policy. The results indicate that decentralization in health surveillance developed slowly and unevenly, which indicates a clear mismatch with the same process in the SUS with regard to their institutional structures, strategies for negotiation and relationship between the spheres. We conclude that the difficulties faced in the decentralization process are also related to tensions arising from different political projects that coexist in these institutional spaces.

KEYWORDS: Decentralization; Unified Health System; Health Surveillance.

\section{RESUMEN:}

En este trabajo se describen las características del proceso de descentralización en el Sistema Único de Salud (SUS) y la vigilancia de la salud, en cuanto a la relación entre los niveles de gestión: la centralización y la descentralización. Se utilizó un enfoque analítico centrado en la relación entre los niveles de gobierno como un factor constitutivo de ejecutar una política social. Los resultados indican que la vigilancia de la salud desarrolló un proceso lento y dessigual de descentralización, que expresa un claro desequilibrio con el mismo proceso en el SUS, en relación con la estructura institucional, las estrategias para la negociación y la relación entre las esferas. Se concluye que las dificultades que enfrentan en el proceso de descentralización también están relacionados con las tensiones que surgen de diferentes proyectos políticos que conviven en estos espacios institucionales.

PALABRAS CLAVE: Descentralización; Sistema de Salud; Vigilancia Sanitaria. 


\section{Introdução}

A criação do Sistema Único de Saúde (SUS), em 1988, implicou a mudança não apenas da forma de organização da rede de saúde no país, mas também dos princípios que a regem, modificando seu conteúdo e lugar no campo das políticas sociais. Desde sua criação, o SUS é orientado por princípios doutrinários - universalidade, equidade e integralidade nos serviços e nas ações de saúde - e por princípios organizativos (ou diretrizes) - descentralização, regionalização e hierarquização da rede e participação social (Brasil, 2000). Nesse período, foram criados mecanismos e instrumentos que visaram ao aprofundamento da descentralização, diretriz que até hoje representa um grande desafio para o Sistema.

Durante as duas décadas de implementação do SUS, a vigilância sanitária sofreu marcantes transformações em seu modelo institucional e de atuação. As principais delas dizem respeito, por um lado, à criação da Agência Nacional de Vigilância Sanitária (Anvisa), em 1999, e, por outro, aos esforços crescentes para a diminuição do distanciamento histórico entre a vigilância sanitária e as práticas de saúde. Entretanto, a despeito dessas iniciativas, até hoje persistem muitas dificuldades para a composição de um arranjo sistêmico para o chamado Sistema Nacional de Vigilância Sanitária - SNVS (Lucchese, 2001b \& De Seta, 2007). Estabelecido legalmente, o SNVS é definido como o conjunto de ações de vigilância sanitária, "executado por instituições da administração pública direta e indireta da União, dos estados, do Distrito Federal e dos municípios, que exerçam atividades de regulação, normatização, controle e fiscalização na área de vigilância sanitária" (Brasil, 1999).

Este trabalho tem por objetivo analisar as características do processo de descentralização no Sistema Único de Saúde e na vigilância sanitária, no que diz respeito aos papéis assumidos pelas três esferas de gestão.

\section{Percurso metodológico}

Segundo Behring e Boschetti (2008), a análise de políticas sociais consiste na compreensão dos aspectos que the imprimem forma e configuração. Para isso, a análise deverá superar o enfoque gerencialista que vem sendo tradicionalmente adotado e permitir um entendimento que leve em conta fundamentalmente o papel e a ação do Estado na formulação e no desenvolvimento das políticas sociais. A partir do reconhecimento do caráter histórico-estrutural das políticas sociais, quatro dimensões perpassam sua análise, de forma complexa e imbricada: história, economia, política e cultura.

Neste estudo, adotou-se a abordagem analítica proposta por Boschetti (2006), que aponta três aspectos para o desenho do quadro institucional que conforma a política a ser analisada: (i) configuração e abrangência dos direitos e benefícios; (ii) configuração do financiamento e gasto; (iii) gestão e controle social. Desse conjunto de aspectos, o terceiro, gestão e controle social, inclui a relação entre as esferas governamentais em seus fatores constitutivos.

A presente análise se concentra nos papéis assumidos pelas três esferas de gestão nas trajetórias de descentralização do SUS e da vigilância sanitária. Os elementos empí- 
ricos utilizados foram: estrutura institucional e competências; estratégias de pactuação, e relação entre as esferas. A Constituição Federal de 1988 e a Lei Orgânica da Saúde estabelecem a descentralização como princípio operativo do SUS, com autonomia das esferas e controle social. Busca-se, a partir do estabelecido legalmente, identificar as características da implantação desse processo, com base nos três elementos empíricos estudados. A análise incluiu revisão bibliográfica e análise de documentos e normativas relacionadas ao processo de descentralização no SUS e na vigilância sanitária.

\section{A descentralização no Sistema Único de Saúde}

\subsection{Estrutura institucional e competências}

A estrutura institucional do SUS está definida na Lei n. 8.080, de 19 de setembro de 1990 , segundo a qual a direção única do Sistema é exercida em cada esfera de governo. Na União, a direção do SUS é realizada pelo Ministério da Saúde; nos estados, Distrito Federal e municípios, pela respectiva secretaria de saúde ou órgão equivalente. Essa Lei estabelece diferentes competências para cada esfera, que devem guiar suas decisões e ações e permitir a sua complementariedade (Brasil, 1990). Em síntese, a direção nacional possui competência para formular, avaliar e apoiar políticas e sistemas em diversas áreas, estabelecer normas, critérios e padrões em vários setores, prestar cooperação técnica e financeira aos estados, ao Distrito Federal e aos municípios e promover a descentralização de serviços e ações de saúde para unidades federadas e municípios. A direção estadual possui competência para promover a descentralização de serviços e ações de saúde para os municípios, acompanhar, controlar e avaliar as redes hierarquizadas do Sistema, coordenar, prestar apoio técnico e financeiro e, em caráter complementar, executar ações e serviços de saúde. A direção municipal possui competência para planejar, organizar, controlar e avaliar as ações e os serviços de saúde e gerir e executar os serviços públicos de saúde.

Na prática, o processo de descentralização do SUS na década de 1990 e, consequentente, a concretização dessas competências foram orientados pelas Normas Operacionais Básicas (NOBs). Nesse período, publicaram-se quatro instrumentos normativos desse tipo - 1991, 1993, 1996 e 1998 -, que representaram a síntese das negociações e dos pactos firmados entre os gestores das três esferas de direção do SUS e do ConseIho Nacional de Saúde. As NOBs trataram de aspectos referentes à divisão de responsabilidades e a relações entre gestores e critérios de transferência de recursos federais para estados e municípios (Levcovitz, Lima, \& Machado, 2001; Brasil, 2007a). De um modo geral, as NOBs estabeleceram critérios que condicionaram o repasse financeiro a determinadas condições de gestão de ações e serviços de saúde, para que os municípios se tornassem responsáveis pela gestão do sistema local de saúde. Em 2004, por meio da Portaria n. 2.023/GM, de 23 de setembro, o Ministério da Saúde estabelece a Gestão Plena de Sistema Municipal como única modalidade de habilitação de municípios, o que consolidou as competências legais previstas para essa esfera no SUS.

\subsection{Estratégias de pactuação}


O uso de indicadores - operacionais e epidemiológicos - foi uma prática adotada desde a criação do SUS e mais marcantemente a partir de 1996, com a criação do Pacto de Indicadores da Atenção Básica. Inicialmente estabelecido como um conjunto de indicadores utilizado para avaliar o desempenho de estados e municípios e a situação de saúde, o Pacto se firmou como instrumento nacional de monitoramento e avaliação de ações e serviços referentes à atenção básica, visando à qualificação da gestão municipal (Bastos, 2009). Criou-se, ainda, a Programação Pactuada Integrada, um instrumento voltado para a organização da assistência e dos recursos, que explicitava competências das três esferas de governo no setor saúde. Nesse momento, a questão da responsabilidade sanitária já era sinalizada como eixo central de um novo pacto federativo na saúde (Oliveira, 2004).

Uma mudança marcante no processo normativo do SUS, com o encerramento do exercício de descentralização tutelada, aconteceu em 2006, com o lançamento do Pacto pela Saúde, um conjunto de diretrizes e compromissos articulados pelos entes federativos em três dimensões: o Pacto pela Vida, o Pacto em Defesa do SUS e o Pacto de Gestão. A sua implementação ocorreu a partir da substituição dos processos de habilitação em condições de gestão pelo Termo de Compromisso de Gestão, que estabelece metas e compromissos para cada ente da federação que a ele adere. Assim é que ficou instaurado o conceito de responsabilidade sanitárias das três esferas na gestão do SUS.

Em 2011, foi publicado o Decreto n. 7.508, que regulamenta a Lei n. 8080/1990 e es- tabelece uma nova dinâmica no sistema de saúde, particularmente no que diz respeito às relações interfederativas. $O$ Decreto estabelece o Contrato Organizativo da Ação Pública da Saúde (Coap) como instrumento de gestão compartilhada e definição de responsabilidades no SUS. A relação contratual entre os gestores, estabelecida a partir de então, destina-se à organização e ao financiamento de ações e serviços de saúde, para garantir a integração dos entes federativos e a integralidade da assistência à saúde dos usuários (Brasil, 2011).

\subsection{Relação entre as esferas}

Segundo Kugelmas e Sola (1999), o regime federativo brasileiro foi marcado por uma alternância entre períodos de centralização e descentralização: um longo período centralizador a partir da Revolução de 1930, que culmina com o Estado Novo; um período democrático da Constituição de 1946; o período do regime militar de 1964, quando a centralização autoritária atinge seu ponto máximo, e a transição democrática e a promulgação da Constituição de 1988, um marco de descentralização federativa. Para os autores, o panorama atual é de indefinição do padrão de relações intergovernamentais, um reflexo do que consideram o "caráter inconcluso da institucionalização democrática no país" ( $p$. 76).

A década de 1990, período de implantação do SUS, e, portanto, de sua diretriz de descentralização, consolida, por um lado, a abertura democrática, mas, por outro, é um período marcado por tensões e obstáculos ao desenvolvimento de políticas sociais. É nesse momento que os governos brasileiros incorporam o ideário neoliberal, caracteriza- 
do, entre outros, pela redução da intervenção do Estado e consequente desmantelamento dos sistemas de proteção social (Diniz, 1996). Viana e Machado (2009) apontam algumas repercussões do predomínio de políticas que priorizam o mercado sobre o processo de descentralização político-administrativo em saúde nessa década. Entre elas, destacam-se a baixa incorporação das especificidades regionais no desenho das estratégias de descentralização e a insuficiente articulação com outras políticas públicas econômicas e sociais. Apesar dessas dificuldades, muitos avanços aconteceram e a descentralização ganhou força, especialmente a partir da última metade dessa década.

Em meio aos conflitos gerados no processo de redefinição dos papéis, o modelo de descentralização caminhou em direção à municipalização da gestão, da explicitação, na esfera federal, da função coordenadora do sistema de referência intermunicipal e do fortalecimento da função indutora e regulatória da esfera federal sobre o processo de descentralização (Levcovitz et al., 2001). Ao mesmo tempo, os espaços de negociação intergestores se consolidaram como instâncias de articulação e pactuação do SUS na esfera federal e nos governos locais - as Comissões Intergestores Tripartite, na direção nacional, e Bipartite, na direção estadual. Instituídas na década de 1990 como espaços privilegiados para negociação e decisão sobre a descentralização das ações e dos serviços do SUS, as Comissões se tornaram, ao longo desse período, "importantes arenas políticas de representação federativa nos processos de formulação e implementação das políticas de saúde" (Brasil, 2007a, p. 53).
Não há consenso sobre se o modelo de federalismo sanitário brasileiro tem um componente predominante de cooperação ou de competição. $O$ fato é que ambos se manifestam na formação do modelo institucional do SUS. A cooperação pode ser observada, por exemplo, nas instâncias permanentes de pactuação, que garantem o caráter de federalismo intraestatal (Brasil, 2006). Entretanto, o componente de competição é verificado no processo de municipalização, muitas vezes "solitário", como apontado por Solla (2006), resultado da dificuldade de assunção de funções e de cooperação entre estados e municípios.

\section{A descentralização na vigilância sanitá- ria}

\subsection{Estrutura institucional e competências}

A discussão sobre a necessidade de implementar serviços descentralizados e articulados em vigilância sanitária remontam à década de 1980, na I Conferência Nacional de Saúde do Consumidor, que aconteceu em 1986. A partir de então, ocorreram várias tentativas de instituir o SNVS, sem sucesso (De Seta, 2007). A Portaria n. 1.565 do Ministério da Saúde, de 26 de agosto de 1994, e a NOB n. 01/1996 apontaram a necessidade de construção de um sistema de atuação estratégica em vigilância sanitária, que envolvesse responsabilidades nas três esferas de governo.

Essa construção foi concretizada na Lei $n$. 9.782/1999 (de criação da Anvisa), que institui o SNVS, definido como o conjunto de ações executado por instituições da Administração Pública direta e indireta da União, dos Estados, do Distrito Federal e dos Mu- 
nicípios, que exerçam atividades de regulação, normatização, controle e fiscalização na área de vigilância sanitária (Brasil, 1999). Segundo a mesma Lei, a coordenação do Sistema, que seria definido a posteriori pela União, compete à Anvisa. O Sistema permanece sem definição formal quanto à sua composição e relação entre as partes. De acordo com definição não formal da Anvisa, que consta no portal eletrônico da Agência, o Sistema inclui:

(...) o Ministério da Saúde, a Agência Nacional de Vigilância Sanitária (Anvisa), o Conselho Nacional de Secretários Estaduais de Saúde (CONASS), o Conselho Nacional de Secretários Municipais de Saúde (CONASEMS), os Centros de Vigilância Sanitária Estaduais, do Distrito Federal e Municipais (VISAS), os Laboratórios Centrais de Saúde Pública (LACENS), o Instituto Nacional de Controle de Qualidade em Saúde (INCQS), a Fundação Oswaldo Cruz (FIOCRUZ), e os Conselhos Estaduais, Distrital e Municipais de Saúde, em relação às ações de vigilância sanitária (Brasil, 2009a).

Entretanto, outros órgãos interagem direta ou indiretamente com essas ações e a falta de contorno desse arranjo é uma primeira dificuldade para considerá-lo como sistema (Lucchese, 2001b). Portanto, se características desse Sistema, tais como composição, estrutura ou operacionalização, não foram objeto de debates antes de sua formalização em lei, tampouco essa definição foi discutida durante a implementação desse arranjo (Lucchese, 2001a).

A Lei n. 9.782/1999 define as competências da União quanto à atuação em vigilância sa- nitária. Em se tratando dos estados e municípios, as competências das esferas de gestão seguem, de um modo geral, a mesma lógica de descentralização estabelecida pela Lei n. 8080/1990. Não houve regulamentação das competências e atribuições de estados e municípios no que diz respeito à vigilância sanitária e, portanto, esse entendimento não foi definido. Isso é problemático porque a vigilância sanitária apresenta características diferentes das ações assistenciais de saúde, às quais as competências dos entes federados são aplicáveis (Lucchese, 2001b).

\subsection{Estratégias de pactuação}

$\mathrm{Na}$ vigilância sanitária, o marco inicial importante de pactuação no SUS ocorreu por meio da NOB n. 01/1996, que estabeleceu duas estratégias de pagamento direto pela execução de ações por parte das secretarias estaduais e municipais de saúde: (i) o Programa Desconcentrado de Ações de Vigilância Sanitária (PDAVS), referente às ações de competência exclusiva da Secretaria de Vigilância Sanitária do Ministério da Saúde; (ii) Ações de Média e Alta Complexidade em Vigilância Sanitária (de competência estadual e municipal).

Apesar do repasse regular de recursos financeiros pelo órgão federal para induzir a execução dessas ações, não houve a correspondente estruturação e o funcionamento de serviço correspondente nos municípios. A execução da maioria das competências de vigilância sanitária permaneceu sob responsabilidade dos serviços estaduais. Mesmo nessas instâncias, a insuficiência e fragilidade do financiamento da vigilância sanitária contribuíam para a sua condição marginal no sistema de saúde (Covem, 2010). 
De 2000 a 2006, vigorou uma forma de pactuação das ações, o Termo de Ajuste e Metas (TAM), mediante a definição de metas organizativas e finalísticas para estados e municípios. Embora tenha contribuído para a estruturação dos órgãos de vigilâncias sanitárias no país, algumas dificuldades foram identificadas ao longo desse período nos mecanismos de pactuação do TAM (Brito, 2007): viés financeiro na implementação em detrimento do compromisso sanitário; repasse tardio dos recursos financeiros aos municípios, apenas em 2004; baixa adesão, especialmente dos municípios, pelo descompasso entre as novas atribuições assumidas pelos gestores e os valores insuficientes de financiamento; pactuação de metas generalistas, incoerentes e não raro desvinculadas do quadro de saúde local; monitoramento precário das ações pactuadas, com baixa articulação entre as esferas na programação, execução e, principalmente, avaliação de resultados, e assimetria de relações e estratégias entre as três esferas de gestão.

Por muito tempo, portanto, o SNVS permaneceu como "um arranjo restrito aos serviços de vigilância sanitária, com baixo grau de coesão entre os componentes federal e estadual, incipiência do componente municipal e precário controle social" (Abrasco, 2009). Uma tentativa de modificar esse quadro aconteceu a partir da implantação do Pacto pela Saúde pelo Ministério da Saúde, quando um novo modelo de pactuação das ações foi adotado pela vigilância sanitária, a partir de 2007.

A mudança principal se refere à adoção do Teto Financeiro de Vigilância Sanitária
(TFVISA), formado por dois pisos. O primeiro, o piso estruturante, é destinado à estruturação e fortalecimento da gestão por meio de elementos, como instalações físicas, equipamentos, sistema de informações, marco legal, capacitação de equipe técnica e instalação de serviço de acolhimento de denúncias. $O$ piso estratégico é composto por ações estratégicas para o gerenciamento do risco sanitário, tais como monitoramento de qualidade de produtos, investigação de surtos e controle de doenças cujos fatores determinantes estejam em seu campo de atuação. Esse conjunto de elementos e ações é pactuado por meio do plano de ação em vigilância sanitária, um instrumento anual de planejamento com a descrição dos resultados esperados e recursos financeiros necessários (Brasil, 2007b; Brasil, 2007c).

Essas mudanças resultaram em avanços para a descentralização das ações de vigilância sanitária no país. Destaca-se o fortalecimento da pactuação nos municípios. Em 2006, o número de municípios com TAM pactuado era 791 , o que corresponde a $14 \%$ do total de municípios do país. Em 2009, existiam 3.131 municípios com planos de ação pactuados com a Comissão Intergestores Bipartite (CIB), o que representa um aumento de quase quatro vezes da participação dessa esfera de gestão na pactuação de vigilância sanitária (Brito, 2007; Covem, 2010). A implementação dos planos de ação resultou em aumento do volume de recursos financeiros alocados e o acompanhamento de sua execução pela Anvisa sinalizou maior visibilidade da vigilância sanitária na gestão de saúde e ampliação no elenco de ações desenvolvidas (Brasil, 2009b).

A partir de 2009, essa normativa foi atua- 
lizada, com a definição de dois pisos para o Componente de Vigilância Sanitária do Bloco de Vigilância em Saúde. O Piso Fixo de Vigilância Sanitária (PFVISA) visa ao fortalecimento do processo de descentralização, à execução das ações de vigilância sanitária e à qualificação das análises laboratoriais de interesse para a vigilância sanitária. O segundo piso, Variável de Vigilância Sanitária, é destinado a incentivos específicos para implementação de estratégias nacionais de interesse da vigilância sanitária, definidas na forma tripartite (Conass, 2014). Os critérios para a manutenção do repasse dos recursos passaram a depender da regularidade na alimentação dos dados nos Sistemas de Informação em Saúde e do detaIhamento das ações de vigilância sanitária, inserido na Programação Anual da Saúde (PAS).

A despeito desses avanços, persistem problemas a serem enfrentados, principalmente quanto à coordenação do SNVS, à gestão de pessoas e ao suporte laboratorial na vigilância sanitária (Ferraro, Costa \& Vieira-da-Silva, 2009; Brasil, 2009b). Assim é que tem sido identificado o risco do plano de ação se tornar um instrumento burocrático, que não assegure o planejamento nem a implementação das ações necessárias ao cumprimento das metas pactuadas (Correa et al., 2009). Além disso, segundo Lucchese (2010), nesse processo, houve apenas a transferência de tarefas para os municípios, sem o devido debate e a formulação de propostas inovadoras de (re)organização de suas ações no plano nacional, o que resultou na continuidade do modus operandi "cartorial e bacharelesco".

Outra dificuldade diz respeito à baixa in- serção da vigilância sanitária na agenda do SUS, tanto nas instâncias formais, quanto nos conselhos de saúde e colegiados gestores, como em outros fóruns estratégicos para a Política Nacional de Saúde (Brito, 2007). Como forma de superar essa limitação, foi criado, em 2002, o Comitê Consultivo de Vigilância Sanitária no âmbito da Comissão Intergestores Tripartite. Durante o período de seu funcionamento, até 2006, o Comitê tratou de temas que ofereceram as bases para a redefinição de diretrizes para o financiamento da vigilância sanitária. No entanto, no mesmo período, houve o esvaziamento político do Comitê (De Seta, 2007), que não conseguiu estabelecer adequada integração técnica e política com o Grupo de Trabalho de Vigilância em Saúde da Comissão Intergestores Tripartite (CIT).

Posteriormente, as funções do Comitê foram transferidas para o Grupo Técnico de Vigilância em Saúde (GTVS) da CIT, mas a representação da vigilância sanitária era minoritária. Isso contribuiu para o enfraquecimento da coordenação federativa exercida pela Anvisa e, portanto, manutenção ou de reforço da separação político-institucional das ações de vigilância sanitária (De Seta \& Dain, 2010). Considerando que os colegiados de gestão funcionam como instância de harmonização, publicização e pactuação dos diferentes interesses presentes nas três esferas de governo (Santos e Merhy, 2006), esse aspecto fica comprometido sem a devida articulação com essas instâncias.

\subsection{Relação entre as esferas}

Durante as décadas de 1970 e 1980, a vigilância sanitária teve a sua gestão marcada por uma característica centralizadora, com 
ações de vigilância de produtos distribuídas entre União e estados, mas sem instrumentos normativos que garantissem a articulação dessas duas esferas. Dessa forma, a Secretaria Nacional de Vigilância Sanitária (SNVS), criada em 1976, atuava de forma esporádica e assistemática junto às secretarias estaduais de saúde. Isso resultou num modelo de vigilância defasado, insuficiente e cartorial (Lucchese, 2001a).

Somente na década de 1990 foram efetivamente implantadas estratégias para a organização de um arranjo nacional de vigilância sanitária (Lucchese, 2001b). Ao mesmo tempo, inicia-se, na SNVS, um processo intenso de desregulamentação, sob o pretexto de busca de maior eficiência e modernização da gestão. Entretanto, as medidas adotadas para esse fim se mostraram voltadas ao interesse do setor privado por meio da desresponsabilização do Estado, provocando o sucateamento da instituição, já bastante precária (Piovesan, 2002).

Esse período foi bastante conturbado para a vigilância sanitária, marcada por denúncias de corrupção na Secretaria, tragédias no setor da saúde relacionadas à fragilidade de atuação da vigilância sanitária e sucessivos episódios de falsificação de medicamentos. Nesse ambiente de crise, o debate sobre a organização nacional de vigilância sanitária foi preterido e as discussões giravam, ao final da década, em torno da necessidade de uma nova autarquia especial para a área, 0 que culminou com a criação da Anvisa, em 1999 (Lucchese, 2001a).

De Seta (2007) tipifica três categorias de constrangimentos à efetivação do SNVS: os decorrentes do pacto federativo pós-1988; os relacionados à descentralização no SUS implementada como municipalização, e os referentes à descentralização na vigilância sanitária. Nesta última, destaca-se a análise de que, após a criação da Anvisa, o modelo de descentralização continuou a favorecer o desequilíbrio de relação entre os entes federados, com reforço predominante à esfera estadual. Além disso, estabeleceu-se também uma assimetria de poder entre os órgãos de vigilância sanitária, com diferentes modelos institucionais e capacidades financeiras. Assim é que, em 2006, já se apontavam problemas como o acompanhamento insuficiente do processo de descentralização nos municípios e uma relação de baixa aproximação entre as esferas (Brito, 2007).

\section{Considerações finais}

Apesar de ser um componente indissociável do SUS, a vigilância sanitária adquiriu, ao longo de sua trajetória, características que a distanciam da Política Nacional de Saúde. Esse distanciamento histórico das práticas de saúde, evidenciado pela escassez de indicadores que expressem a efetividade de suas ações, reforçou a noção de vigilância sanitária como prática centrada apenas no controle, sendo muitas vezes vista como alheia ao conceito de vigilância em saúde pública (Silva Júnior, 2004). As ações de vigilância sanitária nas três esferas, embora representem um espaço privilegiado de promoção e garantia do direito à saúde (Campos, 2009), são marcadas pela baixa vinculação ao SUS.

Essas diferenças ficam evidentes nas trajetórias de descentralização da vigilância sanitária e do SUS. Nos anos de 1990, enquanto normativas e pactuações faziam evo- 
luir a descentralização no SUS, a vigilância sanitária era pautada por modelo centralizador, em meio a graves problemas político-institucionais. Após a criação da Anvisa, em 1999, inicia-se um processo lento e irregular de descentralização, o que expressa um claro descompasso com o mesmo processo no SUS. Nessa época, os municípios já estavam em vias de assumir plenamente a competência de gestão de ações e serviços de saúde, o que ocorreu em 2004.

As estratégias de pactuação também evoluíram de forma diferente no SUS e na vigilância sanitária. Enquanto no primeiro as pactuações caminharam desde o início na direção da responsabilidade dos entes e do monitoramento e da avaliação por meio de indicadores operacionais e epidemiológicos, na vigilância sanitária o caráter financeiro foi predominante por muito tempo, ao lado da fragilidade dos mecanismos de acompanhamento. As estratégias de pactuação da vigilância sanitária, portanto, refletem as dificuldades decorrentes, por um lado, da falta de mecanismos formais e legais da direção do SNVS, e, de outro, da baixa capacidade de avaliação do processo de descentralização nesse campo (Lucchese, 2006).

O Quadro 1 sintetiza a análise das características do processo de descentralização na vigilância sanitária e no SUS, de acordo com as categorias utilizadas.

Quadro 1. Síntese do processo de descentralização do SUS e da vigilância sanitária, segundo categorias analíticas.

\begin{tabular}{|c|c|c|c|}
\hline & $\begin{array}{l}\text { ESTRUTURA INSTITUCIO- } \\
\text { NAL E COMPETÊNCIAS }\end{array}$ & $\begin{array}{l}\text { ESTRATÉGIAS DE PACTUA- } \\
\text { ÇÃO }\end{array}$ & $\begin{array}{c}\text { RELAÇÃO ENTRE AS ESFE- } \\
\text { RAS }\end{array}$ \\
\hline $\begin{array}{l}\text { SISTEMA ÚNICO } \\
\text { DE SAÚDE }\end{array}$ & $\begin{array}{l}\text { Competências estabelecidas na Lei } \\
\text { n. } 8.080 / 1990 . \\
\text { 1990: Definição de responsabilida- } \\
\text { des por meio das NOBs. } \\
\text { 2004: Gestão Plena de Sistema } \\
\text { Municipal. }\end{array}$ & $\begin{array}{l}\text { Descentralização tutelada na década } \\
\text { de } 1990 . \\
\text { Pactos de gestão a partir dos anos } \\
\text { de } 2000 . \\
\text { Contrato Operativo de Saúde Públi- } \\
\text { ca em } 2011 \text {. }\end{array}$ & $\begin{array}{l}\text { Marcado por um modelo misto de } \\
\text { cooperação e competição. }\end{array}$ \\
\hline $\begin{array}{l}\text { VIGILÂNCIA } \\
\text { SANITÁRIA }\end{array}$ & $\begin{array}{l}\text { Definição parcial de competências } \\
\text { na Lei n. 9.782/1999. } \\
\text { Os componentes e a dinâmica do } \\
\text { Sistema não foram explicitamente } \\
\text { definidos. }\end{array}$ & $\begin{array}{l}\text { NOB 01/1996: indução à descentra- } \\
\text { lização de ações } \\
2000 \text { a 2006: metas organizativas } \\
\text { para estados e municípios (Termo } \\
\text { de Ajuste e Metas). } \\
\text { A partir de 2007: Teto Financeiro da } \\
\text { Vigilância Sanitária. }\end{array}$ & $\begin{array}{l}\text { Centralizadora até a década de } \\
1990 . \\
\text { Após 1990, o modelo de descen- } \\
\text { tralização com desequilíbrio de } \\
\text { relação entre os entes federados: } \\
\text { esfera estadual predominante. }\end{array}$ \\
\hline
\end{tabular}

Fonte: Elaboração própria 
Mesmo se levando em conta as dificuldades inerentes ao arranjo federativo (Arretche, 2002) e as especificidades da vigilância sanitária nesse arranjo (De Seta, 2007), ela também se mostra defasada em relação ao SUS quanto à relação entre as esferas. $O$ desencontro na autonomia dos entes federados, a carência de dimensão doutrinária e política e a baixa coesão entre as esferas são alguns dos problemas apontados por Lucchese (2001b) e ainda não resolvidos no SNVS. Assim, a delimitação clara dos objetivos e das diretrizes do SNVS é um dos principais requisitos para a superação dessas dificuldades.

De acordo com Hofling (2001), a relação estabelecida entre Estado e políticas sociais determina as estratégias governamentais adotadas em determinado período histórico: o Estado pode atuar, então, como regulador a serviço da manutenção das relações capitalistas. Dessa forma, o impacto das políticas implementadas é o resultado da tentativa de mediação - pelo Estado - de interesses conflitivos. Nesse caso, o próprio modelo institucional de agência, adotado na vigilância sanitária, diz respeito ao ideário neoliberal, que preconiza a não intervenção estatal, de forma a permitir que o livre mercado se encarregue de equalizar as relações entre os indivíduos. Esse conjunto de contradições impressas na trajetória da vigilância sanitária, além de trazer esses novos desafios, aprofundou o distanciamento histórico com as políticas de saúde. Some-se a isso o fato de o SUS ser o resultado de uma agenda progressista e fruto de mobilização de setores da sociedade, que propôs um modelo contra-hegemônico aos interesses mercantilistas. A reversão desse quadro pressupõe, portanto, não apenas questões relacionadas ao arcabouço normativo e a estratégias de pactuação, mas também as tensões inerentes ao projeto político assumido por cada um desses espaços institucionais.

\section{REFERÊNCIAS}

Abrasco - Associação Brasileira de Pós-graduação em Saúde Coletiva. Grupo Temático de Vigilância Sanitária. Síntese do Relatório da Oficina "Subsídios para Consolidação do Sistema Nacional de Vigilância Sanitária". (2004). Recuperado em 10 dez., 2009, de <http://www.abrasco.org.br/grupos/arquivos/20060717152551.pdf>.

Arretche, M. T. S. (2002). Relações federativas nas políticas sociais. Educação e Sociedade, 80(23), 25-48.

Bastos, M. M. A. (2009). Avaliação da implementação do pacto de indicadores da atenção básica em Secretarias Estaduais de Saúde no Brasil. Dissertação de mestrado em Saúde Pública, Escola Nacional de Saúde Pública Sergio Arouca, Fundação Oswaldo Cruz, Rio de Janeiro, 162 p.

Behring, E. R.; Boschetti, I. (2008). Política social: fundamentos e história. pp. 25-36. São Paulo: Cortez.

Brasil. Agência Nacional de Vigilância Sanitária. Agência/SNVS - Sistema Nacional de Vigilância Sanitária. Recuperado em 10 dez., 2009a, de <http://portal.anvisa.gov.br/ wps/portal/ anvisa/agencia/snvs>.

Brasil. Agência Nacional de Vigilância Sanitária. (2009b). Relatório de Atividades 2008/ Agência Nacional de Vigilância Sanitária. 
Brasília: Anvisa. 133 p.

Brasil. Conselho Nacional de Secretários de Saúde. (2007a). Sistema Único de Saúde (Coleção Progestores - Para entender a gestão do SUS, 1). Brasília: Conass. 291 p.

Brasil. Conselho Nacional de Secretários de Saúde. (2007b). Sistema Único de Saúde (Coleção Progestores - Para entender a gestão do SUS, 7). Brasília: Conass. 132 p.

Brasil. Conselho Nacional de Secretários de Saúde. (2006). SUS: avanços e desafios. Brasília: Conass. 164 p.

Brasil. Decreto n. 7.508, de 28 de junho de 2011. (2011, 29 jun.). Regulamenta a Lei n. 8.080, de 19 de setembro de 1990, para dispor sobre a organização do Sistema Único de Saúde - SUS, o planejamento da saúde, a assistência à saúde e a articulação interfederativa, e dá outras providências. Diário Oficial [da] República Federativa do Brasil, Poder Executivo, Brasília, DF.

Brasil. Lei n. 8.080, de 19 de setembro de 1990. (1990, 20 set.). Dispõe sobre as condições para a promoção, proteção e recuperação da saúde, a organização e o funcionamento dos serviços correspondentes e dá outras providências. Diário Oficial [da] República Federativa do Brasil, Poder Executivo, Brasília, DF.

Brasil. Lei n. 9.782, de 26 de janeiro de 1999. (1999, 11 fev.). Define o Sistema Nacional de Vigilância Sanitária, cria a Agência Nacional de Vigilância Sanitária, e dá outras providências. Diário Oficial [da] República Federativa do Brasil, Poder Executivo, Brasília, DF.
Brasil. Ministério da Saúde. (2011). Contrato Organizativo da Ação de Saúde Pública. Brasília: Ministério da Saúde

Brasil. Ministério da Saúde. (2009c). O SUS no seu munic ípio: garantindo saúde para todos (Série B. Textos Básicos de Saúde). Brasília: Ministério da Saúde. 46 p.

Brasil. Ministério da Saúde. (2000). Sistema Único de Saúde (SUS): princípios e conquistas. Brasília: Ministério da Saúde. 44 p.

Brasil. Portaria 1.998, de 21 de agosto de 2007. (2007c, 22 ago.). Regulamenta o repasse de recursos financeiros destinados à execução das ações de vigilância sanitária na forma do Componente de Vigilância Sanitária do Bloco de Financiamento de Vigilância em Saúde. Diário Oficial [da] República Federativa do Brasil, Poder Executivo, Brasília, DF.

Boschetti, I. (2006). Dimensões, categorias e indicadores para análise e avaliação de políticas sociais. Texto didático preparado para a disciplina Análise de Políticas Sociais. Programa de Pós-graduação em Política Social. SER-UnB.

Brito, R. L. (2009). A construção do Plano Diretor de Vigilância Sanitária: um processo inovador de planejamento, pactuação e gestão no âmbito do SUS. Revista Ciência e Saúde Coletiva, v.14, supl.esp.

Brito, R. L. (2007). Análise da Política de Descentralização das Ações de Vigilância Sanitária no Brasil: do debate sobre o repasse de recursos ao compromisso com a responsabilidade sanitária. 206p. Disserta- 
ção de mestrado em Saúde Pública, Centro de Pesquisas Aggeu Magalhães, Fundação Oswaldo Cruz, Recife.

Campos, G. W. S. Vigilância Sanitária: responsabilidade pública na proteção e promoção da saúde. p. 10. Recuperado em 12 dez. 2009, de <http://www.anvisa.gov.br/divulga/ conavisa/cadernos/eixo1_texto01.pdf $>$.

Conass - Conselho Nacional de Secretários de Saúde. (2013). Nota Técnica 25/2013 - financiamento de vigilância sanitária. Recuperado em 25 jun., 2014, de <http://www.conass.org.br/Notas\%20t\%C3\%A9cnicas $\% 20$ 2013/NT\%2025\%20-\%20Financiamento $\% 20 \mathrm{de} \% 20$ VISA.pdf $>$.

Correa, C. B. et al. (2009). Plano de ação de vigilância da saúde: instrumento para planejamento ou instrumento burocrático? Ciência e Saúde Coletiva, 14(supl.esp).

Covem, E. M. (2010). Descentralização das ações de vigilância sanitária. Ciência \& Saúde Coletiva, Rio de Janeiro, 15(supl. 3), 3318-3328.

De Seta, M. H. (2007). A Construção do Sistema Nacional de Vigilância Sanitária: uma análise das relações intergovernamentais na perspectiva do federalismo. $188 \mathrm{p}$. Tese de doutorado em Saúde Coletiva, Instituto de Medicina Social, Universidade do Estado do Rio de Janeiro, Rio de Janeiro.

De Seta, M. H., \& Dain, S. (2010). Construção do Sistema Brasileiro de Vigilância Sanitária: argumentos para debate. Ciência e Saúde Coletiva, 15(supl. 3), 3307-3317.

Diniz, E. (1996). Em busca de um novo pa- radigma: a reforma do Estado no Brasil dos anos 90. São Paulo em Perspectiva, 10(4), 13-26.

Ferraro, A. H. A., Costa, E. A., \& Vieira-da-Silva, L. M. (2009). Imagem-objetivo para a descentralização da vigilância sanitária em nível municipal. Cadernos de Saúde Pública, 25(10), 2201-2217.

Hofling, E. M. (2001). Estado e políticas (públicas) sociais. Cadernos Cedes, 21(55), 30-41.

Kugelmas, E., \& Sola, L. (1999). Recentralização/Descentralização: dinâmica do regime federativo no Brasil dos anos 90. Revista de Sociologia da Universidade de São Paulo, 11(2), 63-81.

Levcovitz, E., Lima, L. D., \& Machado, C. V. (2001). Política de saúde nos anos 90: relações intergovernamentais e o papel das Normas Operacionais Básicas. Ciência e Saúde Coletiva, 6(2), 269-291.

Lucchese, G. (2001a). A Vigilância Sanitária no Sistema Único de Saúde. In: Conferência Nacional de Vigilância Sanitária, 1, pp. 5369, Brasília, DF. Caderno de textos. Brasília: Agência Nacional de Vigilância Sanitária.

Lucchese, G. (2010). Descentralização e modelo sistêmico: o caso da vigilância sanitária. Ciência e Saúde Coletiva, 15(supl. 3), 3318-3328.

Lucchese, G. (2001b). Globalização e regulação sanitária. Os rumos da vigilância sanitária no Brasil. 2001. 245 p. Tese de doutorado em Saúde Pública, Escola Nacional de Saúde Pública, Fundação Oswaldo Cruz, 
Rio de Janeiro.

Lucchese, G. (2006). A vigilância sanitária no Sistema Único de Saúde. In: De Seta, M. H.; Pepe, V. L. E., \& Oliveira, G. O. Gestão e Vigilância Sanitária: modos atuais do pensar e fazer. pp. 33-48. Rio de Janeiro: Editora Fiocruz.

Oliveira, F. J. F. O. (2004). A Programação Pactuada e Integrada (PPI) no contexto da Norma de Assistência à Saúde (NOAS) e o Conselho Nacional de Saúde: Uma discussão sobre a gestão do SUS. 155p. Dissertação de mestrado em Gestão de Sistemas e Serviços da Saúde, Escola Nacional de Saúde Pública Sergio Arouca, Fundação Oswaldo Cruz, Rio de Janeiro.

Piovesan, M. F. (2002). Construção Política da Agência Nacional de Vigilância Sanitária. 108 p. Dissertação de mestrado em Saúde Pública, Escola Nacional de Saúde Pública, Fundação Oswaldo Cruz, Rio de Janeiro.
Santos, F. P., \& Merhy, E. E. (2006). A regulação pública da saúde no Estado brasileiro uma revisão. Interface - Comunicação, Saúde, Educação, 9(18), 25-41.

Silva J., J. B. (2004). Epidemiologia em Serviço: uma avaliação de desempenho do Sistema Nacional de Vigilância em Saúde. 318 p. Tese de doutorado em Saúde Coletiva, Universidade Estadual de Campinas, São Paulo, SP.

Solla, J. J. S. P. (2006). Avanços e limites da descentralização no SUS e o "Pacto de Gestão." Revista Baiana de Saúde Pública, 30(2), 332-348.

Viana, A. L. A., \& MACHADO, C. V. (2009). Descentralização e coordenação federativa: a experiência brasileira na saúde. Ciência \& Saúde Coletiva, Rio de Janeiro, 14(3), 807817. 